\title{
A Fuzzy Inventory Model for Vendor - Buyer Coordination in a Two Stage Supply Chain with Allowed Shortages
}

\author{
P. Parvathi \\ Head \& Associate Professor, \\ Department of Mathematics, \\ Quaid- E -Millath Government College for \\ Women (Autonomous), \\ Chennai-600002
}

\author{
D. Chitra \\ Assistant Professor, \\ Department of Mathematics, \\ Quaid- E -Millath Government College for \\ Women (Autonomous), \\ Chennai-600002
}

\begin{abstract}
This paper develops Fuzzy inventory model to determine the relevant profit maximizing decision variable values. The model proposed is based on individual profits of vendor and buyer and joint total profit of them which finds out optimal ordering quantity, selling price and shipment policies. Shortages are allowed for both of them. Production rate, ordering quantity, shortage and holding cost of buyer and vendor are taken as triangular fuzzy numbers. Graded mean integration representation method is used for defuzzification. The conclusion drawn from Numerical example is, it is more beneficial for the buyer and vendor to co-operate with each other when the demand is more selling price sensitive.
\end{abstract}

\section{Keywords}

Price sensitive demand, shipments, vendor-buyer coordination, fuzzy concepts.

\section{INTRODUCTION}

Production and marketing are the two major functions of every business that involves manufacturing and selling. Inventory is a part of these major factors. Production is the process of converting raw materials into finished products. Marketing is the process of meeting the end needs of buyer. The business scenario may vary with buyer and vendor. Sometimes the vendor who manufactures the product may fall shortage due to breakdown of machines to supply for buyer which in turn is a shortage of buyer. Shortages refer to inability to meet the demand at the required time schedule as preferred by the customer.

To overcome this situation, there should be better coordination and more co-operations between the vendor and buyer so as to earn more profit. If no co-ordination exists, the supply chain members act independently to maximize their own profits. In traditional inventory management the inventory and shipment polices for the vendor and the buyer in a two echelon chain are managed independently. The optimal lot size for the buyer may not result in an optimal policy for the vendor and vice versa.

To overcome this difficultly, the integrated vendor - buyer model has been developed using fuzzy costs where the joint total relevant cost for both buyer and vendor is minimized. Determining the ordering and shipment polices results in a reduction of the total inventory costs of the system if the determination is based on integrated total cost function rather than buyer's, vendor's individual cost function.

Goyal (3) early developed the idea of a joint total cost for a single vendor and single buyer scenario assuming an infinite production rate for the vendor and lot-for-lot policy for the shipments from the vendor to the buyer. Goyal (4) introduced a model where the shipment size increases by a factor equal to the ratio of the production rate to the demand rate. He formulated the problem and developed an optimal expression for the first shipment size as a function of number of shipments.

Lau and Lau [5] framed a joint-pricing inventory model with out setup cost. Ray, et al.,[6] introduced a integrated marketing inventory model for two pricing policies, price as a decision variable and mark-up pricing. R. Akbari jokar [7] developed joint model to determine the profit function of buyer and vendor.

A. Nagoor Gani and G. Sabarinathan [8] developed fuzzy integrated inventory model to determine the relevant profit maximizing decision variable values. They did not allow shortages which is unrealistic. To suit the real life situation this paper allows shortages for both vendor and buyer. The final demand for the product is assumed to be deterministic but price sensitive. Production rate, ordering quantity, setup cost, shortage cost and holding cost of the buyer and vendor are taken as triangular fuzzy numbers. The lots delivered from the vendor to the buyer are equal - sized batches. As soon as the on-hand inventory at the buyer drops to reorder point, an order of size $\tilde{y}$ is released by the buyer. The vendor manufactures the product at the production rate $\tilde{P}$ and in lot sizes which are a multiple of $\tilde{y}$. The objective is to determine the number of shipments, the selling price $\tilde{\delta}$ as well as order size by allowing shortages for buyer and vendor, so that the total profit of the vendor - buyer is maximized.

\section{METHODOLOGY}

\subsection{Fuzzy Numbers}

Any fuzzy subset of the real line R, whose membership function $\mu_{\mathrm{A}}$ satisfied the following conditions, is a generalized fuzzy number $\tilde{A}$. 
(i) $\mu_{\mathrm{A}}$ is a continuous mapping from $\mathrm{R}$ to the closed interval $[0,1]$,

(ii) $\mu_{\mathrm{A}}=0,-\infty<x \leq a_{1}$

(iii) $\mu_{\mathrm{A}}=\mathrm{L}(\mathrm{x})$ is strictly increasing on $\left[\mathrm{a}_{1}, \mathrm{a}_{2}\right]$

(iv) $\mu_{\mathrm{A}}=\mathrm{w}_{\mathrm{A}}, a_{2} \leq x \leq a_{3}$

(v) $\mu_{\mathrm{A}}=\mathrm{R}(\mathrm{x})$ is strictly decreasing on $\left[\mathrm{a}_{3}, \mathrm{a}_{4}\right]$

(vi) $\mu_{\mathrm{A}}=0, a_{4} \leq x \leq \infty$

where $0<\mathrm{w}_{\mathrm{A}} \leq 1$ and $\mathrm{a}_{1}, \mathrm{a}_{2}, \mathrm{a}_{3}$ and $\mathrm{a}_{4}$ are real numbers. Also this type of generalized fuzzy number be denoted as $\widetilde{A}=\left(a_{1}, a_{2}, a_{3}, a_{4} ; w_{A}\right)_{L R}$; when $\mathrm{w}_{\mathrm{A}}=1$, it can be simplified as $\tilde{A}=\left(a_{1}, a_{2}, a_{3}, a_{4} ; w_{A}\right)_{L R}$.

\section{Triangular fuzzy number}

The fuzzy set $\tilde{A}=\left(a_{1}, a_{2}, a_{3}\right)$ where $\mathrm{a}_{1}<\mathrm{a}_{2}<\mathrm{a}_{3}$ and defined on

$\mathrm{R}$, is called the triangular fuzzy number, if the membership function of $A$ is given by

$\mu_{A}=\left\{\begin{array}{l}\frac{x-a_{1}}{a_{2}-a_{1}}, a_{1} \leq x \leq a_{2} \\ \frac{a_{3}-x}{a_{3}-a_{2}}, a_{2} \leq x \leq a_{3} \\ 0, \text { otherwise }\end{array}\right.$

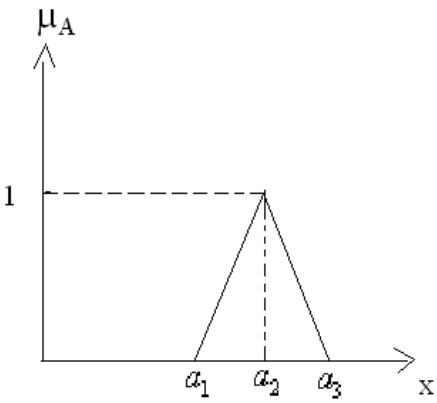

\section{The Function Principle}

The function principle was introduced by Chen [6] to treat fuzzy arithmetical operations. This principle is used for addition, subtraction, multiplication and division of fuzzy numbers.

$$
\text { Suppose } \quad \tilde{A}=\left(a_{1}, a_{2}, a_{3}\right) \quad \text { and }
$$

$\widetilde{B}=\left(b_{1}, b_{2}, b_{3}\right)$ are two triangular fuzzy numbers. Then

(i) The addition of $\tilde{A}$ and $\tilde{B}$ is

$$
\widetilde{A}+\widetilde{B}=\left(a_{1}+b_{1}, a_{2}+b_{2}, a_{3}+b_{3}\right) \text { where }
$$

$a_{1}, a_{2}, a_{3}, b_{1}, b_{2}, b_{3}$ are any real numbers.

(ii) The multiplication of $\tilde{A}$ and $\tilde{B}$ is $\widetilde{A} \times \widetilde{B}=\left(c_{1}, c_{2}, c_{3}\right)$

where

$T=\left(a_{1} b_{1}, a_{1} b_{3}, a_{3} b_{1}, a_{3} b_{3}\right), c_{1}=\min T, c_{2}=a_{2} b_{2}, c_{3}=\max T$ if $\mathrm{a}_{1}, \mathrm{a}_{2}, \mathrm{a}_{3}, \mathrm{~b}_{1}, \mathrm{~b}_{2}, \mathrm{~b}_{3}$ are all non zero positive real numbers, then $\widetilde{A} \times \widetilde{B}=\left(a_{1} b_{1}, a_{2} b_{2}, a_{3} b_{3}\right)$.

(iii) $-\widetilde{B}=\left(-b_{3},-b_{2},-b_{1}\right)$ then the subtraction of $\widetilde{B}$ from $\tilde{A} \quad$ is $\quad \tilde{A}-\widetilde{B}=\left(a_{1}-b_{3}, a_{2}-b_{2}, a_{3}-b_{1}\right)$ where $a_{1}, a_{2}, a_{3}, b_{1}, b_{2}, b_{3}$ are any real numbers.

(iv) $\frac{1}{\widetilde{B}}=\tilde{B}^{-1}=\left(\frac{1}{b_{3}}, \frac{1}{b_{2}}, \frac{1}{b_{1}}\right)$ where $\mathrm{b}_{1}, \mathrm{~b}_{2}, \mathrm{~b}_{3}$ are all non zero positive real numbers, then the division of $\tilde{A}$ and $\tilde{B}$ is $\frac{\widetilde{A}}{\widetilde{B}}=\left(\frac{a_{1}}{b_{3}}, \frac{a_{2}}{b_{2}}, \frac{a_{3}}{b_{1}}\right)$

(v) For any real number $\mathrm{K}$, $K \tilde{A}=\left(K a_{1}, K a_{2}, K a_{3}\right)$ if $K>0$

$K \tilde{A}=\left(K a_{3}, K a_{2}, K a_{1}\right) i f K<0$

Graded Mean Integration Representation Method

$$
\text { If } \widetilde{A}=\left(a_{1}, a_{2}, a_{3}, a_{4} ; w_{A}\right)_{L R} ; \text { is a }
$$

generalized fuzzy number then the defuzzified value $\mathrm{P}(\tilde{A})$ by graded mean integration representation method is given

by $p(\tilde{A})=\frac{\int_{0}^{w_{a}} h\left[\frac{L^{-1}(h)+R^{-1}(h)}{2}\right] d h}{\int_{0}^{w_{A} 1} h d h}$,

with $0<\mathrm{h} \leq \mathrm{w}_{\mathrm{A}}$ and $0<\mathrm{w}_{\mathrm{A}} \leq 1$. If $\tilde{A}=\left(a_{1}, a_{2}, a_{3}\right)$ is a triangular number then the graded mean integration representation of $\tilde{A}$ by above formula is

$p(A)=\frac{1}{2}\left[\frac{\int_{0}^{1} h\left[a_{1}+h\left(a_{2}-a_{1}\right)+a_{3}-h\left(a_{3}-a_{2}\right)\right] d h}{\int_{0}^{1} h d h}\right]$ $=\frac{a_{1}+4 a_{2}+a_{3}}{6}$ 


\section{Notations}

\begin{tabular}{|c|c|c|}
\hline $\mathrm{P}$ & - & Production rate of the vendor \\
\hline $\mathrm{y}$ & - & Order quantity of the buyer \\
\hline $\mathrm{K}_{\mathrm{v}}$ & - & Setup cost of the vendor \\
\hline $\mathrm{K}_{\mathrm{b}}$ & - & Ordering cost of the buyer \\
\hline $\mathrm{c}$ & - & the buyer's unit purchasing price \\
\hline$\delta$ & - & Unit selling price of the buyer \\
\hline $\begin{array}{l}\mathrm{D} \\
\text { selling pri }\end{array}$ & - & Demand rate as a function of unit \\
\hline $\begin{array}{l}\mathrm{h}_{\mathrm{v}} \\
\text { per year }\end{array}$ & - & inventory holding cost for the vendor \\
\hline $\begin{array}{l}\mathrm{h}_{\mathrm{b}} \\
\text { year }\end{array}$ & - & inventory holding cost for the buyer per \\
\hline $\mathrm{n}$ & - & Number of shipments \\
\hline $\mathrm{S}_{\mathrm{b}}$ & - & Shortage cost for the buyer \\
\hline $\mathrm{S}$ & - & Maximum inventory level of the buyer \\
\hline $\begin{array}{l}S_{1} \\
\text { vendor }\end{array}$ & - & Maximum inventory level of the \\
\hline
\end{tabular}

$\widetilde{P}=\left(P_{1}, P_{2}, P_{3}\right) \quad-\quad$ Fuzzy production rate of the vendor

$\tilde{y}=\left(y_{1}, y_{2}, y_{3}\right)$

Fuzzy order

quantity of the buyer

$\tilde{K}_{v}=\left(K_{v_{1}}, K_{v_{2}}, K_{v_{3}}\right) \quad$ - $\quad$ Fuzzy setup cost of the vendor

$$
\tilde{K}_{b}=\left(K_{b_{1}}, K_{b_{2}}, K_{b_{3}}\right) \quad \text { - } \quad \text { Fuzzy ordering cost }
$$

of the buyer

$\widetilde{\delta}=\left(\delta_{1}, \delta_{2}, \delta_{3}\right)$

Fuzzy unit selling

price of the buyer

$$
\tilde{D}=\left(D_{1}, D_{2}, D_{3}\right)
$$

Fuzzy demand rate as a function of unit selling price

$$
\tilde{h}_{v}=\left(h_{v_{1}}, h_{v_{2}}, h_{v_{3}}\right)
$$

Fuzzy inventory holding cost for the vendor per year

$$
\tilde{h}_{b}=\left(h_{b_{1}}, h_{b_{2}}, h_{b_{3}}\right) \quad-\quad \text { Fuzzy inventory }
$$

holding cost for the buyer per year

$$
\tilde{n}=\left(n_{1}, n_{2}, n_{3}\right)
$$

Number

of

shipments

$$
\tilde{S}_{b}=\left(S_{b_{1}}, S_{b_{2}}, S_{b_{3}}\right) \quad-\quad \text { Fuzzy inventory }
$$

shortage cost for the buyer per unit per unit time

$$
\tilde{S}_{v}=\left(S_{v_{1}}, S_{v_{2}}, S_{v_{3}}\right) \quad-\quad \text { Fuzzy inventory }
$$

shortage cost for the vendor per production per unit time
$T \tilde{P}_{v}$

Annual

profit

function for the vendor

$T \tilde{P}_{B}$

Annual

profit function for the buyer

\section{Assumptions}

(i) The model deals with a single vendor and a single buyer for a single product.

(ii) The buyer faces a linear Demand $\tilde{D}(\tilde{\delta})$ as a function of selling price $\tilde{\delta}$.

(iii) The inventory is continuously reviewed. The buyer orders a lot of size $\tilde{y}$ when the on-hand inventory reaches the reorder point.

(iv) The vendor manufactures a production batch $n \tilde{y}$ at one setup. However, the size of shipment delivered to the buyer is $\tilde{y}$.

(v) The inventory holding cost at the buyer is higher than that at the vendor. i.e., $\tilde{h}_{b}>\tilde{h}_{v}$

(vi) Shortages are allowed for both vendor and buyer.

(vii) The time horizon is infinite.

\section{FUZZY MATHEMATICAL MODEL}

The optimal policy of the integrated system is derived. However, for comparative purposes, we first obtain the buyer and the vendor policies, if each party optimizes its profit independently. The policies and profits are then compared to the case of integrated system when they co-operate, particularly in information sharing.

Assume that the buyer faces a linear demand $\tilde{D}(\tilde{\delta})=a-b \tilde{\delta}(\mathrm{a}>\mathrm{b}>0)$ as a function of its unit selling price. As $\tilde{D}(\tilde{\delta})>0$, the maximum selling price is a/b, i.e., $\widetilde{\delta}<\frac{a}{b}$. The buyer wishes to maximize his yearly profit function, $T \tilde{P}_{B}$ through the optimal choice of selling price and order quantity, i.e.,

$$
\begin{aligned}
\tilde{T} P_{B}(\tilde{\delta}, \tilde{y})= & (a-b \tilde{\delta})(\tilde{\delta}-c) \\
& -\frac{\tilde{h}_{b} \tilde{S}^{2}}{2 \tilde{y}}-\frac{\tilde{S}_{b}(\tilde{Q}-\tilde{S})^{2}}{2 \tilde{y}} \\
& -\frac{\tilde{K}_{b}(a-b \tilde{\delta})}{\tilde{y}}
\end{aligned}
$$

where

$$
\tilde{y}=\sqrt{2 \tilde{K}_{b}(a-b \tilde{\delta}) \frac{\tilde{h}_{b}+\tilde{S}_{b}}{\tilde{h}_{b} \tilde{S}_{b}}}
$$




$$
\tilde{S}=\frac{\tilde{y} \tilde{S}_{b}}{\tilde{h}_{b}+\tilde{S}_{b}}
$$

substituting equation (3) in equation (1), we get,

$$
\begin{aligned}
\tilde{T} P_{B}(\tilde{\delta}, \tilde{y})= & (a-b \tilde{\delta})(\tilde{\delta}-c) \\
& -\frac{\tilde{h}_{b} \tilde{S}_{b}{ }^{2} \tilde{y}}{2\left(\tilde{h}_{b}+\tilde{S}_{b}\right)^{2}} \\
& -\frac{\tilde{S}_{b} \tilde{y}\left(1-\frac{\tilde{S}_{b}}{\tilde{h}_{b}+\tilde{S}_{b}}\right)^{2}}{2} \\
& -\frac{\tilde{K}_{b}(a-b \tilde{\delta})}{\tilde{y}}
\end{aligned}
$$

Substituting equation (2) in (1) we get,

$T \tilde{P}_{B}(\tilde{\delta})=\left(a \tilde{\delta}-b \tilde{\delta}^{2}-a c+b c \tilde{\delta}\right)-$

$\sqrt{(a-b \tilde{\delta})}\left\{\begin{array}{l}\frac{\tilde{h}_{b} \tilde{S}_{b}^{2} \sqrt{2 \tilde{K}_{b}} \sqrt{\frac{\tilde{h}_{b}+\tilde{S}_{b}}{\tilde{h}_{b} \tilde{S}_{b}}}}{2\left(\tilde{h}_{b}+\tilde{S}_{b}\right)^{2}} \\ +\frac{\tilde{S}_{b}\left(1-\frac{\tilde{S}_{b}}{\tilde{h}_{b}+\tilde{S}_{b}}\right)^{2} \sqrt{2 \tilde{K}_{b}} \sqrt{\frac{\tilde{h}_{b}+\tilde{S}_{b}}{\tilde{h}_{b} \tilde{S}_{b}}}}{2} \\ +\frac{\tilde{K}_{b}}{\sqrt{2 \tilde{K}_{b}} \sqrt{\frac{\tilde{h}_{b}+\tilde{S}_{b}}{\tilde{h}_{b} \tilde{S}_{b}}}}\end{array}\right\}$

Differentiating the above equation with respect to $\tilde{\delta}$,

$$
\left.\begin{array}{rl}
\frac{\partial T \tilde{P}_{B}(\tilde{\delta})}{\partial \tilde{\delta}}=a & -2 b \tilde{\delta}+b c+ \\
\frac{b}{2 \sqrt{(a-b \tilde{\delta})}}\left\{\begin{array}{l}
\frac{\tilde{h}_{b} \tilde{S}_{b}^{2} \sqrt{2 \tilde{K}_{b}} \sqrt{\frac{\tilde{h}_{b}+\tilde{S}_{b}}{\tilde{h}_{b} \tilde{S}_{b}}}}{2\left(\tilde{h}_{b}+\tilde{S}_{b}\right)^{2}} \\
+\frac{\tilde{S}_{b}\left(1-\frac{\tilde{S}_{b}}{\tilde{h}_{b}+\tilde{S}_{b}}\right)^{2} \sqrt{2 \tilde{K}_{b}} \sqrt{\frac{\tilde{h}_{b}+\tilde{S}_{b}}{\tilde{h}_{b} \tilde{S}_{b}}}}{2}
\end{array}\right\}---(6) \\
+\frac{\tilde{K}_{b}}{\sqrt{2 \tilde{K}_{b}} \sqrt{\frac{\tilde{h}_{b}+\tilde{S}_{b}}{\tilde{h}_{b} \tilde{S}_{b}}}}
\end{array}\right\}
$$

Differentiating the above equation again with respect to

$\tilde{\delta}$

$\frac{\partial^{2} T \tilde{P}_{B}(\tilde{\delta})}{\partial \tilde{\delta}^{2}}=-2 b+$

$\frac{b^{2}}{4\left(a-b \tilde{\delta}^{\frac{3}{2}}\right.}\left\{\begin{array}{l}\frac{\tilde{h}_{b} \tilde{S}_{b}^{2} \sqrt{2 \tilde{K}_{b}} \sqrt{\frac{\tilde{h}_{b}+\tilde{S}_{b}}{\tilde{h}_{b} \tilde{S}_{b}}}}{2\left(\tilde{h}_{b}+\tilde{S}_{b}\right)^{2}} \\ +\frac{\tilde{S}_{b}\left(1-\frac{\tilde{S}_{b}}{\tilde{h}_{b}+\tilde{S}_{b}}\right)^{2} \sqrt{2 \tilde{K}_{b}} \sqrt{\frac{\tilde{h}_{b}+\tilde{S}_{b}}{\tilde{h}_{b} \tilde{S}_{b}}}}{2}\end{array}\right\}---(7)$

Equating (6) to zero and solving the equation leads to the value of $\tilde{\delta}$, substituting this value of $\tilde{\delta}$ in equation (2) value of $\tilde{y} \cdot *$ is obtained. Now $T \widetilde{P}_{B}(\tilde{\delta})$ is concave.

\section{Vendor's Profit Policy}

When the buyer's order quantity and the selling price are adopted, the orders are received by the vendor at a known interval $\tilde{Q} / \tilde{D}(\tilde{\delta})$.

A vendor's average inventory can then be obtained as follows:

$$
A \tilde{I}_{v}=\frac{\tilde{Q}}{2}\left[(\tilde{n}-1)\left(1-\frac{\tilde{D}(\tilde{\delta})}{\tilde{P}}\right)+\frac{\tilde{D}(\tilde{\delta})}{\tilde{P}}\right]---
$$

Hence the vendor's yearly profit function is ,

$$
\tilde{T} P_{v}(\tilde{n})=a c-b c \tilde{\delta}-\frac{(a-b \tilde{\delta}) \tilde{K}_{v}}{\tilde{n} \tilde{y}}-\frac{\tilde{h}_{v} S^{2}{ }_{1}}{2 \tilde{n} \tilde{y}}-
$$

$$
\frac{\tilde{S}_{v}\left(\tilde{y}-S_{1}\right)^{2}}{2 \tilde{y}}\left[\begin{array}{c}
\tilde{n}\left(1-\frac{(a-b \tilde{\delta})}{\tilde{P}}\right)-1 \\
+\frac{2(a-b \tilde{\delta})}{\tilde{P}}
\end{array}\right]-(9)
$$

such that $\mathrm{n}$ is integer.

$$
S_{1}=\frac{\tilde{y} \tilde{S}_{v}}{\tilde{S}_{v}+\tilde{h}_{v}}------(10)
$$

Substituting (10) in (9), 


$$
\begin{aligned}
& \tilde{T} P_{v}(\tilde{n})=a c-b c \tilde{\delta}-\frac{(a-b \tilde{\delta}) \tilde{K}_{v}}{\tilde{n} \tilde{y}} \\
& -\frac{\tilde{h}}{2 \tilde{y}}\left(\frac{\tilde{S}_{v}}{\tilde{S}_{v}+\tilde{h}_{v}}\right)^{2}- \\
& \frac{\tilde{S}_{v} \tilde{y}}{2}\left(1-\frac{\tilde{S}_{v}}{\tilde{S}_{v}+\tilde{h}_{v}}\right)^{2}\left[\begin{array}{l}
\tilde{n}\left(1-\frac{(a-b \tilde{\delta})}{\tilde{P}}\right)-1 \\
+\frac{2(a-b \tilde{\delta})}{\tilde{P}}
\end{array}\right]--(11)
\end{aligned}
$$

First derivative of (9) with respect to $\tilde{n}$,

$$
\begin{aligned}
& \frac{\partial \tilde{T} P_{v}(\tilde{n})}{\partial \tilde{n}}=\frac{(a-b \tilde{\delta}) \tilde{K}_{v}}{\tilde{n}^{2} \tilde{y}}+\frac{\tilde{h}_{v} \tilde{y}}{2 \tilde{n}^{2}}\left(\frac{\tilde{S}_{v}}{\tilde{S}_{v}+\tilde{h}_{v}}\right)^{2}- \\
& \frac{\tilde{S}_{v} \tilde{y}}{2}\left(1-\frac{\tilde{S}_{v}}{\tilde{S}_{v}+\tilde{h}_{v}}\right)^{2}\left[\left(1-\frac{(a-b \tilde{\delta})}{\tilde{P}}\right)\right]--(12)
\end{aligned}
$$

$T \widetilde{P}_{v}(\tilde{n})$ is concave in $\tilde{n}$.

$$
\begin{aligned}
& \frac{\partial \tilde{T} P_{v}(\tilde{n})}{\partial \tilde{n}}=0 \Rightarrow \\
& \tilde{n}^{*}=\sqrt{\frac{\frac{(a-b \tilde{\delta}) \tilde{K}_{v}}{\tilde{y}}+\frac{\tilde{h}_{v} \tilde{y}}{2}\left(\frac{\tilde{S}_{v}}{\tilde{S}_{v}+\tilde{h}_{v}}\right)^{2}}{\frac{\tilde{S}_{v} \tilde{y}}{2}\left(1-\frac{\tilde{S}_{v}}{\tilde{S}_{v}+\tilde{h}_{v}}\right)^{2}\left[\left(1-\frac{(a-b \tilde{\delta})}{\tilde{P}}\right)\right]}}--(13)
\end{aligned}
$$

\section{INTEGRATED MODEL POLICY}

If the buyer is free to choose his own marketing and ordering policies $(\tilde{\delta}, \tilde{Q})$, and the vendor is free to choose his number of shipments $\mathrm{n}$, then it is straight forward that the total system profit under individual optimization, $T \widetilde{P}_{1}(\tilde{\delta}, \tilde{Q}, \tilde{n})$ is equal to the sum of buyer's and the vendor's profits.

$$
T \tilde{P}_{1}(\tilde{\delta}, \tilde{Q}, \tilde{n})=T \tilde{P}_{B}(\tilde{\delta})+T \tilde{P}_{v}(\tilde{n})
$$

Suppose that both parties decide to cooperate and agree to follow the joint optimal integrated policy. The cost stemming from the purchasing price is an internal transfer of money from one supply chain member (the vendor) to another supply chain member (the buyer).
Therefore it is not a cost of the whole supply chain. The total system profit under joint optimization with shortage is,

$$
\begin{aligned}
& T P_{j}(\tilde{y}, \widetilde{\delta}, \widetilde{n})=\left\{\begin{array}{l}
(a-b \widetilde{\delta})(\widetilde{\delta}-c) \\
-\frac{\widetilde{h}_{b} \widetilde{S}_{b}^{2} \tilde{y}}{2\left(\widetilde{h}_{b}+\widetilde{S}_{b}\right)^{2}} \\
-\frac{\widetilde{S}_{b} \tilde{y}\left(1-\frac{\widetilde{S}_{b}}{\widetilde{h}_{b}+\widetilde{S}_{b}}\right)^{2}}{2}-\frac{\widetilde{K}_{b}(a-b \tilde{\delta})}{\tilde{y}}
\end{array}\right\}+
\end{aligned}
$$

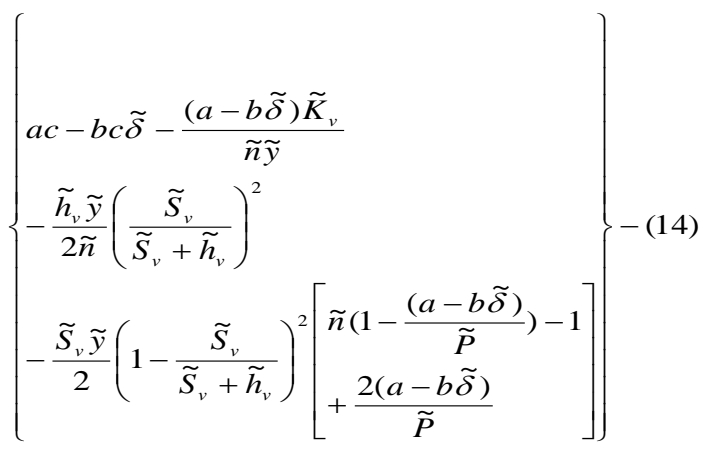

Differentiating with respect to $\tilde{y}$

$$
\begin{aligned}
& \frac{\partial T P_{j}}{\partial \tilde{y}}=(a-b \tilde{\delta}) \frac{\left(\tilde{K}_{b}+\frac{\tilde{K}_{v}}{\tilde{n}}\right)}{\tilde{y}^{2}} \\
& -\left[\begin{array}{l}
\frac{\tilde{h}_{b}}{2}\left(\frac{\tilde{S}_{b}}{\left(\tilde{h}_{b}+\tilde{S}_{b}\right)}\right)^{2} \\
+\frac{\tilde{S}_{b}\left(1-\frac{\tilde{S}_{b}}{\tilde{h}_{b}+\tilde{S}_{b}}\right)^{2}}{2} \\
+\frac{\tilde{h}_{v}}{2 \tilde{n}}\left(\frac{\tilde{S}_{v}}{\tilde{S}_{v}+\tilde{h}_{v}}\right)^{2} \\
+\frac{\tilde{S}_{v}}{2}\left(1-\frac{\tilde{S}_{v}}{\tilde{S}_{v}+\tilde{h}_{v}}\right)^{2}\left[\begin{array}{l}
\tilde{n}\left(1-\frac{(a-b \tilde{\delta})}{\tilde{P}}\right) \\
-1 \\
+\frac{2(a-b \tilde{\delta})}{\tilde{P}}
\end{array}\right]
\end{array}\right]
\end{aligned}
$$

Differentiating the above equation partially with respect $\tilde{y}$ and equating this derivative to zero we get 


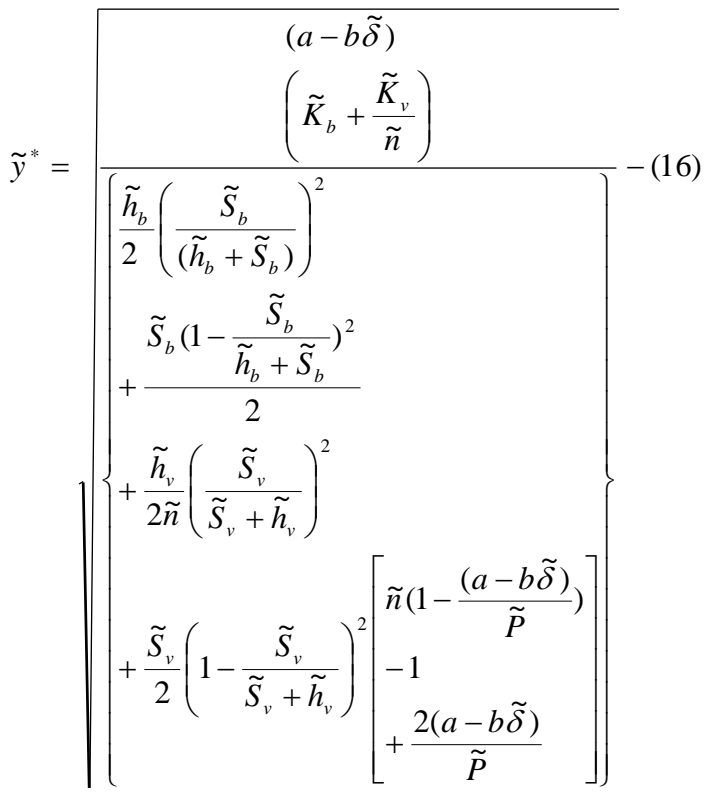

Substituting (16) in (14) we get,

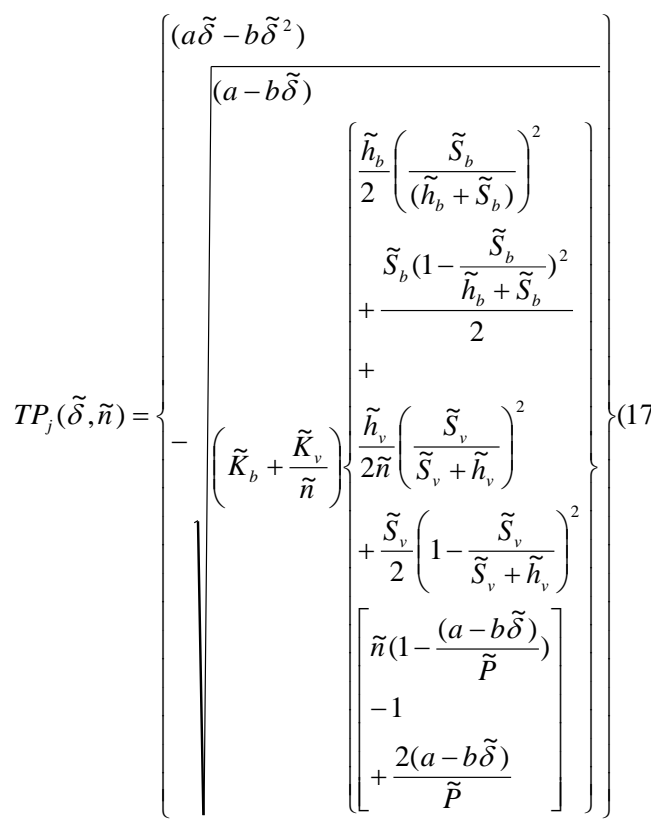

Let

$$
T P_{j}^{1}(\tilde{\delta}, \tilde{n})=(a-b \tilde{\delta})\left(\tilde{K}_{b}+\frac{\tilde{K}_{v}}{\tilde{n}}\right)\left\{\begin{array}{l}
\left.\tilde{h}_{b}\left(\frac{\tilde{S}_{b}}{2}\right)^{2}+\tilde{S}_{b}\right) \\
+\frac{\tilde{S}_{b}\left(1-\frac{\tilde{S}_{b}}{\tilde{h}_{b}+\tilde{S}_{b}}\right)^{2}}{2} \\
\left.+\frac{\tilde{h}_{v}\left(\frac{\tilde{S}_{v}}{2 \tilde{n}}\right)^{2}}{\tilde{S}_{v}+\tilde{h}_{v}}\right)^{\tilde{S}_{v}\left(\frac { \tilde { S } _ { v } } { 2 } \left(1-\frac{\tilde{S}_{v}+\tilde{h}_{v}}{2}\right.\right.} \\
+\left[\begin{array}{l}
\tilde{n}\left(1-\frac{(a-b \tilde{\delta})}{\tilde{P}}\right) \\
-1+\frac{2(a-b \tilde{\delta})}{\tilde{P}}
\end{array}\right.
\end{array}\right\}(18)
$$

For a given value of $\tilde{\delta}$, maximizing $T \tilde{P}_{j}$ is equivalent to minimizing $T P^{1}{ }_{j}$.

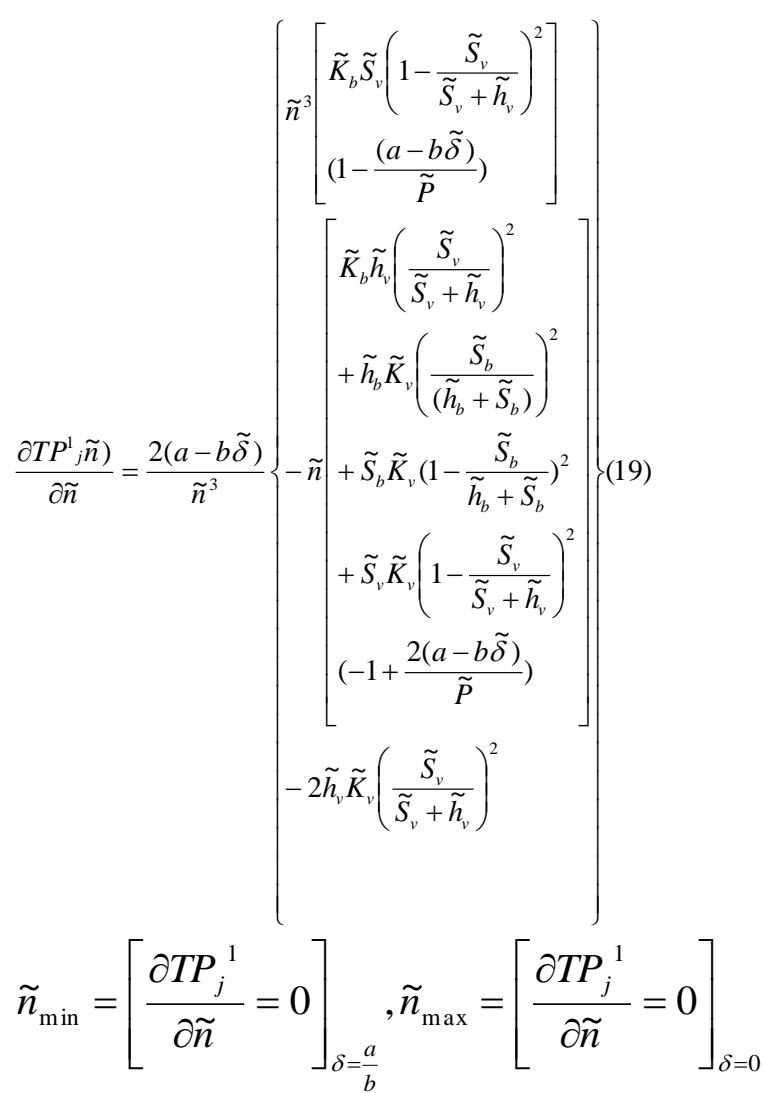

second derivative of $T P^{1}{ }_{j}$ with respect to $\tilde{n}$ is convex. 


\section{DEFUZZIFICATION OF THIS MODEL}

Using Graded mean integration representation method, we will get the crisp value of Selling price $(\delta)$, Order quantity $(\mathrm{Q})$, number of shipments (n) for individual and joint model, Total profit for buyer $\left(\mathrm{TP}_{\mathrm{B}}\right)$, Total profit for vendor $\left(\mathrm{TP}_{\mathrm{v}}\right)$, Total system profit under individual optimization $\left(\mathrm{TP}_{1}\right)$, the joint total profit of vendor $\left(\mathrm{TP}_{\mathrm{vj}}\right)$, the joint total profit of buyer $\left(\mathrm{TP}_{\mathrm{Bj}}\right)$, total system profit under joint optimization $\left(\mathrm{TP}_{\mathrm{j}}\right)$.

For a given value of $n, \mathrm{TP}_{\mathrm{j}}$ can be written as

$T P_{j}(D)=m_{1} D+m_{2} D^{2}-\sqrt{m_{3} D+m_{4} D^{2}}$

where

$$
\begin{aligned}
& m_{1}=\frac{a}{b} \\
& m_{2}=\frac{-1}{b}
\end{aligned}
$$$$
m_{3}=2\left(K_{b}+\frac{K_{v}}{n}\right)\left\{\begin{array}{l}
h_{b}\left(\frac{S_{b}}{\left(h_{b}+S_{b}\right)}\right)^{2} \\
+\frac{h_{v}\left(\frac{S_{v}}{S_{v}+h_{v}}\right)^{2}}{+S_{b}\left(1-\frac{S_{b}}{h_{b}+S_{b}}\right)^{2}} \\
-S_{v}\left(1-\frac{S_{v}}{h_{v}+S_{v}}\right)^{2}(n-1)
\end{array}\right\}
$$$$
m_{4}=\frac{2}{P}\left(K_{b}+\frac{K_{v}}{n}\right) S_{v}\left(1-\frac{S_{v}}{S_{v}+h_{v}}\right)^{2}(2-n)
$$

and $\mathrm{D}(\delta)=\mathrm{a}-\mathrm{b} \delta$

There is a one to one relationship between price and demand. Therefore, we base our analysis on the identification of the optimal value of demand, rather than the optimal value of price. The first and second partial derivative of $\mathrm{TP}_{\mathrm{j}}(\mathrm{D})$, with respect to $\mathrm{D}$ are as follows.

$$
\begin{aligned}
& \frac{\partial T P_{j}(D)}{\partial D}=m_{1}+2 m_{2} D-\frac{m_{3}+2 m_{4} D}{2 \sqrt{m_{3} D+m_{4} D^{2}}} \\
& \frac{\partial^{2} T P_{j}(D)}{\partial D^{2}}=2 m_{2}+\frac{m_{3}^{2}}{4}\left(m_{3} D+m_{4} D^{2}\right)^{\frac{-3}{2}}
\end{aligned}
$$

\section{Case 1: $\mathbf{n}=1$}

Hence $\mathrm{m}_{4}>0$, therefore there are two saddle points, $\mathrm{SP}_{1}$ and $\mathrm{SP}_{2}$. The total profit function is convex when $\mathrm{SP}_{1}<\mathrm{D}$ $<\mathrm{SP}_{2}$, and is concave when $\mathrm{D} \leq \mathrm{SP}_{1}$ or $\mathrm{D} \geq \mathrm{SP}_{2}$. The optimal value of the demand is then

$\mathrm{D}^{*}=\mathrm{LO}_{1}$ if $\mathrm{LO}_{1}<\mathrm{a}$, and it is $\mathrm{D}^{*}=\mathrm{a}$ if $\mathrm{LO}_{1} \geq \mathrm{a}$.

\section{Case 2: $n=2$}

Hence $\mathrm{m}_{4}=0$, and therefore there is a saddle point, $S P=\frac{b^{\frac{2}{3}} m^{\frac{1}{3}} 3}{4}$ the total profit function is convex when D $<\mathrm{SP}$, and is concave when $\mathrm{D} \geq \mathrm{SP}$. Because the total profit function is zero at $\mathrm{D}=0$, there is no more than one local optimal amount for the demand. The optimal value of the demand is then

$\mathrm{D}^{*}=\mathrm{LO}_{2}$ if $\mathrm{LO}_{2}<\mathrm{a}$, and it is $\mathrm{D}^{*}=\mathrm{a}$ if $\mathrm{LO}_{2} \geq \mathrm{a}$.

\section{Case 3: $n \geq 3$}

Hence, $\mathrm{m}_{4}<0$, and therefore there are two saddle points, $\mathrm{SP}_{1}$ and $\mathrm{SP}_{2}$. The total profit function is concave when $\mathrm{SP}_{2}$ $<\mathrm{D}<\mathrm{SP}_{1}$, and it is convex when $\mathrm{D} \leq \mathrm{SP}_{2}$ or $\mathrm{D} \geq \mathrm{SP}_{1}$. Moreover, $\mathrm{m}_{3}, \mathrm{t}>0$ and thus $\mathrm{SP}_{1}>0$ and $\mathrm{SP}_{2}>0$ The optimal value of the demand is then

$\mathrm{D}^{*}=\mathrm{LO}_{3}$ if $\mathrm{LO}_{3}<\mathrm{a}$, and it is $\mathrm{D}^{*}=\mathrm{a}$ if $\mathrm{LO}_{3} \geq \mathrm{a}$.

As no closed form solution exists for the local optimal values of the demand, we use numerical method to find $\mathrm{LO}_{\mathrm{i}}, \mathrm{i}=1,2,3$, . 


\section{NUMERICAL EXAMPLE}

We consider an example with the following data:

$\widetilde{P}=(3100,3200,3300) /$ year

$\tilde{K}_{v}=(R s .300, R s .400, R s .500) /$ setup

$\tilde{K}_{b}=(R s .20, R s .25, R s .30) /$ order

$\tilde{h}_{v}=(R s .3, R s .4, R s .5) /$ unit/ setup

$\tilde{h}_{b}=(R s .4, R s .5, R s .6) /$ unit/ year $\mathrm{a}=1500, \mathrm{~b}=10, \mathrm{c}=$ Rs.5/unit

$$
\tilde{S}_{v}=(R s .80, R s .85, R s .90) / \text { unit }
$$

This paper analyzes the effect demand's price sensitivity. The effect is evaluated by the impact on the benefits of vendor-buyer coordination as well as impact on the decision variables.

$\mathrm{TP}_{\mathrm{j}}$ and $\mathrm{TP}_{1}$ represent the total system profit under joint and individual optimization.

Joint total profit allocated to the buyer and the vendor as follows (see Ouyang et. al., [9], $\mathrm{Wu}$ and Ouyang [10],

$$
T P_{v j}=\frac{T P_{v}(n)}{T P_{1}} T P_{j} a d T P_{B j}=\frac{T P_{B}(n)}{T P_{1}} T P_{j}
$$

$\tilde{S}_{b}=(R s .8, R s .9, R s .10) /$ unit

\begin{tabular}{|c|c|c|c|c|c|c|}
\hline \multicolumn{7}{|c|}{ Decision variables under individual optimization (fuzzy environment) } \\
\hline B & $\delta$ & $\mathrm{Y}$ & $\mathrm{n}$ & $\mathrm{TP}_{\mathrm{v}}$ & $\mathrm{TP}_{\mathrm{B}}$ & $\mathrm{TP}_{1}$ \\
\hline \multirow{3}{*}{10} & $(77.3647$, & $(69.397$, & & $(1724.5$, & $(51507.994$, & $(53232.494$, \\
& 77.5463, & 85.088, & $(5,5,5)$ & 2859.69, & 52162.444, & 55022.134, \\
& $77.7284)$ & $104.102)$ & & $3064.72)$ & $52636.900)$ & $55701.62)$ \\
\hline \multirow{3}{*}{20} & $(39.9611$, & $(74.854$, & & $(1936.24$, & $(23722.09$, & $(25658.33$, \\
& 39.9519, & 104.235, & $(5,5,5)$ & 2898.84, & 24164.34, & 27063.17, \\
& $39.9807)$ & $144.994)$ & & $3128.05)$ & $24371.02)$ & $27499.07)$ \\
\hline \multirow{3}{*}{30} & $(19.2655$, & $(85.885$, & & $(2843.01$, & $(12276.79$, & $(15119.79$, \\
& 19.2656, & 119.547, & $(5,5,5)$ & 3917.35, & 12768.42, & 16685.77, \\
& $19.2657)$ & $166.316)$ & & $4184.20)$ & $12989.61)$ & $17173.90)$ \\
\hline \multirow{3}{*}{40} & $(12.8106$, & $(88.883$, & & $(3119.81$, & 6806.10, & $(9925.91$, \\
& 12.8118, & 123.720, & $(5,5,5)$ & 4210.01, & 7316.01, & 11526.03, \\
& $12.8119)$ & $172.126)$ & & $4498.82)$ & $7545.28)$ & $12044.10)$ \\
\hline
\end{tabular}

\begin{tabular}{|c|c|c|c|c|c|c|}
\hline \multicolumn{7}{|c|}{ Decision variables under joint optimization (fuzzy environment) } \\
\hline $\mathrm{B}$ & $\delta$ & $\mathrm{Y}$ & $\mathrm{n}$ & $\mathrm{TP}_{\mathrm{vj}}$ & $\mathrm{TP}_{\mathrm{Bj}}$ & $\mathrm{TP}_{\mathrm{j}}$ \\
\hline \multirow{3}{*}{10} & $(75.3986$, & $(117.106$, & & $(1686.638$, & $(50377.128$, & $(54478.682$, \\
& 75.4894, & 208.462, & $(4,4,4)$ & 2876.945, & 52477.192, & 58354.138, \\
& $75.6997)$ & $326.682)$ & & $3219.442)$ & $55294.276)$ & $55919.939)$ \\
\hline \multirow{3}{*}{20} & $(37.9611$, & $(99.104$, & & $(1862.52$, & $(22818.99$, & $(26452.19$, \\
& 37.9519, & 201.119, & $(4,4,4)$ & 2962.81, & 24697.58, & 27660.39, \\
& $38.9807)$ & $270.046)$ & & $3480.31)$ & $26301.74)$ & $28547.79)$ \\
\hline \multirow{3}{*}{30} & $(18.2625$, & $(128.3838$, & & $(2640.90$, & $(11403.30$, & $(15953.01$, \\
& 18.2656, & 190.6403, & $(4,4,4)$ & 3936.06, & 12829.24, & 16765.204, \\
& $19.2656)$ & $268.506)$ & & $4959.17)$ & $15288.49)$ & $17919.83)$ \\
\hline \multirow{3}{*}{40} & $(11.8106$, & $(121.167$, & & $(2759.92$, & $(6020.98$, & $(10654.74$, \\
& 11.8118, & 196.100, & $(4,4,4)$ & 4194.02, & 7288.22, & 11482.24, \\
& $12.8118)$ & $260.864)$ & & $5361.04)$ & $9651.58)$ & $12696.77)$ \\
\hline
\end{tabular}




\begin{tabular}{|c|c|c|c|c|c|c|}
\hline \multicolumn{7}{|c|}{ Decision variables under individual optimization (after defuzzification) } \\
\hline $\mathrm{B}$ & $\delta$ & $\mathrm{Y}$ & $\mathrm{n}$ & $\mathrm{TP}_{\mathrm{v}}$ & $\mathrm{TP}_{\mathrm{B}}$ & $\mathrm{TP}_{1}$ \\
\hline 10 & 77.5464 & 85.642 & 5 & 2704.663 & 52132.445 & 54837.108 \\
\hline 20 & 39.9584 & 106.13 & 5 & 2776.61 & 36187.61 & 26901.68 \\
\hline 30 & 19.2656 & 121.17 & 5 & 3782.78 & 19085.02 & 16506.13 \\
\hline 40 & 12.8116 & 125.98 & 5 & 4076.35 & 10903.81 & 11345.69 \\
\hline
\end{tabular}

\begin{tabular}{|c|c|c|c|c|c|c|}
\hline \multicolumn{7}{|c|}{ Decision variables under joint optimization (after defuzzification) } \\
\hline $\mathrm{B}$ & $\delta$ & $\mathrm{Y}$ & $\mathrm{n}$ & $\mathrm{TP}_{\mathrm{vj}}$ & $\mathrm{TP}_{\mathrm{Bj}}$ & $\mathrm{TP}_{\mathrm{j}}$ \\
\hline 10 & 75.50 & 212.94 & 4 & 2735.6 & 52596.695 & 55302.529 \\
\hline 20 & 38.124 & 195.605 & 4 & 2865.68 & 24651.46 & 27606.99 \\
\hline 30 & 18.432 & 193.24 & 4 & 3890.72 & 13001.43 & 16822.27 \\
\hline 40 & 11.978 & 194.40 & 4 & 4149.51 & 7470.90 & 11546.74 \\
\hline
\end{tabular}

\section{CONCLUSION}

This paper has developed a fuzzy integrated production inventory- marketing model for two stage supply chains with shortages. Here it finds the optimal values of ordering quantity, pricing and shipment policies and its crisp values. Even though shortages are allowed for vendor and buyer it gives maximum profit than A. Nagoor Gani., G. Sabarinathan [8].

\section{REFERENCES}

[1] Inventory systems by Eliezer Naddor

[2] Fuzzy sets and logics by Zadeh

[3] Goyal, S.K., 1976. An integrated inventory model for a single supplier-single customer problem. International journal of Production Research 15(1), 107-111.

[4] Goyal, S.K., 1988. A joint economic-lotsize model for purchaser and vendor; a comment Decision science 19, 236-241.

[5] Lau, A.H.L., Lau, H.S., 2003. Effects of a demand-curve's shape on the optimal solutions of a multi-echelon inventory/pricing model. European journal of Operational Research 147, 530-548.

[6] Ray. S. Gerchek. Y., Jewkers. E. M., 2005. Join pricing and inventory policies for make - to - stock products with deterministic price sensitive demand. International Journal of Production Economics 97, 143-158.

[7] Mohsen S. Sajadieh., Mohammad R. Akbari Jokar., 2009. Optimizing shipment, ordering and pricing policies in a two-stage supply chain with price-sensitive demand. Transportation Research Part E 45, 564-571.

[8] A. Nagoor Gani, G. Sabarinathan., 2012. A new method for solving two stage supply chain fuzzy inventory problem., applied mathematical sciences, 60, 2963-2978

[9] Ouyang, L., Wu., K., Ho, C., 2004. Integrated vendor buyer co operating models with stochastic demand in controllable lead time International Journal of Production Economics 92, 255-256.

[10] Wu. k. Ouyang, L., 2003 An integrated single vendor single buyer inventory system with shortage derived algebraically, Production Planning and Control 14(6), 555-561 